\title{
A network pharmacology approach for investigating the multi- target mechanisms of Huangqi in the treatment of colorectal cancer
}

\author{
Xiao-Dong Chu ${ }^{1 \#}$, Yi-Ran Zhang ${ }^{1 \#}$, Zheng-Bin Lin ${ }^{1}$, Zhan Zhao ${ }^{1}$, Shu-Chen Huangfu ${ }^{1}$, Sheng-Hui Qiu ${ }^{1}$, \\ Yan-Guan Guo ${ }^{1}$, Hui Ding ${ }^{1}$, Ting Huang ${ }^{2}$, Xiao-Li Chu ${ }^{3,4}$, Jing-Hua Pan ${ }^{1}$, Yun-Long Pan ${ }^{1}$ \\ ${ }^{1}$ Department of General Surgery, the First Affiliated Hospital of Jinan University, Guangzhou, China; ${ }^{2}$ Department of Clinical Pathology, the First \\ Affiliated Hospital of Jinan University, Guangzhou, China; ${ }^{3}$ School of Economics and Management, Xidian University, Xi'an, China; ${ }^{4}$ Center of \\ Network, Guangdong AIB Polytechnic, Guangzhou, China \\ Contributions: (I) Conception and design: XD Chu, YR Zhang, JH Pan, YL Pan; (II) Administrative support: XD Chu, YL Pan, JH Pan; (III) \\ Provision of study materials or patients: SH Qiu, YG Guo, H Ding, T Huang; (IV) Collection and assembly of data: XD Chu, YR Zhang, ZB Lin, Z \\ Zhao, SC Huangfu; (V) Data analysis and interpretation: XD Chu, YR Zhang, XL Chu; (VI) Manuscript writing: All authors; (VII) Final approval of \\ manuscript: All authors. \\ "These authors contributed equally to this work. \\ Correspondence to: Dr. Jing-Hua Pan, MD, PhD; Prof. Yun-Long Pan, PhD. Department of General Surgery, the First Affiliated Hospital of Jinan \\ University, 613 Huangpu West Avenue, Guangzhou 510632, China. Email: huajanve@foxmail.com; tpanyl@jnu.edu.cn.
}

Background: Colorectal cancer (CRC) is the third most prevalent cancer globally. In the treatment of CRC, surgical resection is commonly adopted, and neoadjuvant chemotherapy or immunotherapy is mainly administered for patients with advanced disease. However, despite the developments in the field of cancer treatment, the mortality rate of CRC has remained high. Therefore, novel treatments for CRC need to be explored. Astragalus membranaceus, commonly known in China as Huangqi (HQ), a traditional Chinese medicine, has been reported to be a potential antitumorigenic agent. This study aimed to investigate the mechanisms of action of $\mathrm{HQ}$.

Methods: Active ingredients and putative targets of $\mathrm{HQ}$ were obtained through a comprehensive search of the Traditional Chinese Medicine Systems Pharmacology database. CRC-related targets were retrieved from the GeneCards database and then overlapping targets were acquired. After visualization of the compound-disease network and protein-protein interaction (PPI) network, Gene Ontology (GO) and Kyoto Encyclopedia of Genes and Genomes (KEGG) enrichment analyses of the overlapping genes were performed. Additionally, HCT116 cells were treated with the active components of HQ at a $20-\mu M$ concentration. Cell Counting Kit-8 was used to detect cell activity, and real-time quantitative polymerase chain reaction was carried out to detect the expression of genes downstream of the interleukin (IL)-17 signaling pathway.

Results: A PPI network comprising 177 nodes and 318 edges was obtained. The GO analysis of the overlapping genes showed enrichment in response to lipopolysaccharide and oxidative process. For the KEGG analysis, the AGE-RAGE signaling pathway and inflammation-related pathways, such as the IL-17 and tumor necrosis factor (TNF) signaling pathways, were enriched. The in vitro experiments showed that HQ promoted the apoptosis of CRC cells by inhibiting the expression of the CCL2, CXCL8, CXCL10, and PTGS2 genes.

Conclusions: This study systematically revealed the multitarget mechanism of HQ in CRC through a network pharmacology approach. We verified that HQ promotes CRC cell death via the IL-17 signaling pathway. This finding provides indications for further mechanistic studies and the development of HQ as a

$\wedge$ ORCID: 0000-0001-6515-8026. 
potential treatment for CRC patients.

Keywords: Huangqi; colorectal cancer (CRC); network pharmacology; multitarget effects

Submitted Jul 24, 2020. Accepted for publication Dec 24, 2020.

doi: $10.21037 /$ tcr-20-2596

View this article at: http://dx.doi.org/10.21037/tcr-20-2596

\section{Introduction}

Colorectal cancer (CRC), a noncommunicable disease, is the third most prevalent cancer in the world, accounting for approximately $10 \%$ of cancer-related fatalities $(1,2)$. Aging, poor dietary habits, smoking, low physical activity, and obesity are the major causes of CRC (3). The proximal colon is the site most commonly affected by CRC (accounting for approximately $41 \%$ of cases), followed by the rectum (28\%), and the distal colon (22\%) (4). Laparoscopic surgery is widely adopted in the treatment of primary CRC, and resection accompanied by radiotherapy is commonly used to treat metastatic disease. For patients with advanced CRC, neoadjuvant and palliative chemotherapy is widely used (5-7). Despite the improvement of treatment strategies for CRC, the mortality rate remains between $40-50 \%$ (2). Therefore, further exploration of novel treatments for CRC patients is necessary.

Traditional Chinese medicine (TCM), an essential component of complementary and alternative therapy, is a product of more than a thousand years of development and is popular in Asian countries, especially China. With their "multicomponent-multitarget-multipathway" characteristics, TCMs have clear advantages in the prevention and treatment of complex diseases, especially in the aspect of "treating the same disease with different treatments" (8). In the treatment of cancer in particular, long-term chemoradiotherapy results in adverse reactions, such as myelosuppression, cumulative neuropathy, gastrointestinal tract reactions, and organ damage, which severely reduce the quality of life of patients and can lead to treatment interruption. Additionally, chemoradiotherapy is often hindered by therapeutic resistance (9). In recent decades, TCM has been used increasingly in Western countries owing to its reduced side effects and its capacity to both prevent and treat cancer (10).

Astragalus membranaceus, colloquially referred to as Huangqi (HQ), is a classical Chinese medicine commonly used to treat cold, diarrhea, fatigue, and anorexia $(11,12)$. It can also play an important role in the treatment of heart failure and chronic liver disease $(8,13)$. Recently, HQ has been shown to inhibit tumor growth and to decrease cell invasiveness and angiogenesis, and it is also capable of enhancing the chemosensitivity of hepatoma cells (14-16). As HQ injection has achieved positive results in clinical trials, it has been approved by the China Food and Drug Administration (17). A series of recent retrospective experimental studies have shown that conventional chemotherapeutics based on FOLFOX 4, 5-FU, and oxaliplatin can be used in combination with HQ and other Chinese herbal medicines to treat mid- to advanced-stage CRC. It can improve the quality of life of patients showing that increases the tumor response rate (TRR) and reduce the incidence of severe neutropenia, nausea, vomiting, and neurotoxicity associated with chemotherapy, showing that HQ has a good antitumor activity in the treatment of CRC $(9,18,19)$. Nevertheless, the ingredients and targets of HQ in CRC need to be further explored.

It is generally believed that drug discovery requires a system-level multi-pharmacological approach to solve problems, such as the lack of efficacy of a singletarget compound and emerging drug resistance. The advancement of the information age has yielded innovations in various network technologies. Based on systems biology, bioinformatics, and high-throughput histology, network pharmacology that integrates pharmacology and information networks is gaining momentum (20). The concept of network pharmacology was first proposed by Hopkins in 2007 (21). It is an effective method for mapping the unexplored target space of natural products, which provides a systematic means to expand the available drug space of proteins related to various complex diseases. By taking a network pharmacology approach, researchers can directly identify drugs and disease targets from a large amount of data (22), and gain a better understanding of the mechanisms and pathways between them, which supports the discovery of new treatment options and readjustments to the use of approved drugs (23). Currently, the scope of application of network pharmacology is expanding. It currently includes exploration of the basic pharmacological 
mechanisms and effects of drugs on diseases, the analysis of TCM theory (24), and study of TCM's application (25). In this network pharmacology analysis, we aimed to investigate the underlying mechanism of HQ administration in the management of CRC at the molecular level, as well as to provide direction for further research in this field. We present the following article in accordance with the MDAR checklist (available at http://dx.doi.org/10.21037/tcr-20-2596).

\section{Methods}

The study was conducted in accordance with the Declaration of Helsinki (as revised in 2013). This research does not involve human subjects, human materials, and therefore is not subject to approval of an institutional ethics committee.

\section{Retrieval of chemical ingredients}

We performed a comprehensive search of the Traditional Chinese Medicine Systems Pharmacology database (TCMSP, https://tcmspw.com/tcmsp.php) to obtain the chemical ingredients of $A$. membranaceus by using the keyword "Huangqi". After retrieving the chemical ingredients, PubChem (https://pubchem.ncbi.nlm.nih. gov/) was used to verify the corresponding compound name and molecular structure. Active compounds of HQ were screened under the criteria of oral bioavailability (OB) $\geq 30 \%$ and drug-likeness (DL) $\geq 0.18$.

\section{Prediction of CRC and HQ targets}

Potential targets of active compounds in HQ were retrieved from the TCMSP database, and their molecular names were converted into gene ID using the Uniprot database (https://www.uniprot.org/). GeneCards is a searchable, integrated database of human genes that provides concise genomic information. We searched for CRC-related genes and targets by using the keyword "colorectal cancer". Then, we used venny (https://bioinfogp.cnb.csic.es/tools/venny/) to visualize the overlapping genes and targets between HQ and CRC for downstream analysis.

\section{Construction of HQ-CRC target network}

Cytoscape (version 3.2.1) was used to construct the compound-disease network. Potential active compounds, genes, and targets of HQ and CRC were treated as the components of the network. The network analysis function of Cytoscape was used to analyze the network topological parameters and to assess the significance of each node according to betweenness centrality (BC) and degree.

\section{Protein-protein interaction (PPI) network}

Overlapping genes and targets of HQ and CRC were input into the Search Tool for the Retrieval of Interacting Genes/ Proteins (STRING) database (https://string-db.org/) to construct the PPI network under the criteria of minimum required interaction score $=0.4$. Disconnected nodes in the network were hidden.

\section{Gene Ontology (GO) and Kyoto Encyclopedia of Genes and Genomes (KEGG) enrichment analysis}

Overlapping genes between HQ and CRC were subjected to GO and KEGG enrichment analyses. The Database for Annotation, Visualization, and Integrated Discovery (DAVID, version 6.8, https://david.ncifcrf.gov/) was used as the reference database.

\section{Cell culture and Cell Counting Kit-8 (CCK-8) assay to detect cell viability}

The human CRC HCT116 cell line was purchased from the American Type Culture Collection and placed in highglycemic Dulbecco's Modified Eagle Medium (DMEM; Guangzhou Genio Biotech Co., Ltd.) containing 1\% penicillin/streptomycin and $10 \%$ fetal bovine serum. The cells were placed in an incubator at a constant temperature of $37^{\circ} \mathrm{C}$ with $5 \% \mathrm{CO}_{2}$, and the medium was changed every 2 days. The cells were observed under a microscope, and $0.25 \%$ trypsin (Guangzhou Genio Biotech Co., Ltd.) was used after the cells are $80-90 \%$ fused. The cells were digested and subculture was continued in a 1:2 ratio. Then, HCT116 cells in good growth condition were inoculated into 96-well plates at $5 \times 10^{3}$ cells/well after trypsinization $(100 \mu \mathrm{L}$ per well), and routinely cultured in an incubator for 24 hours at $37{ }^{\circ} \mathrm{C}$ with $5 \% \mathrm{CO}_{2}$. Quercetin, isorhamnetin, and formononetin (Sigma-Aldrich, USA) were added as the experimental group at a concentration of $20 \mu \mathrm{M}$. Cells treated with dimethyl sulfoxide (DMSO) solution served as a control group. Each group was set up with 5 multiple wells. After culture for 48 hours, the cells were collected, and $10 \mu \mathrm{L}$ CCK-8 reagent (Promega, USA) was added to each well. The cells were incubated at $37^{\circ} \mathrm{C}$ for 2 hours, 
and then a microplate reader was used to determine the absorbance value (A value) at $450 \mathrm{~nm}$. The experiment was repeated 3 times. The results were expressed in terms of cell viability according to the following formula: cell viability = (experimental absorbance - blank hole absorbance)/(control hole absorbance - blank hole absorbance) $\times 100 \%$.

\section{Real-time quantitative polymerase chain reaction (PCR)}

Four primers, CCL2, CXCL8, CXCL10, and PTGS2, were purchased from Sangon Biotech Corporation (Shanghai, China). The sequence details are listed in Table S1. HCT116 cells were intervened with the above 3 drugs at a concentration of $20 \mu \mathrm{M}$ for 48 hours. After that, the cells were collected, and RNA was extracted using the TRIzol method. After the purity and integrity of the RNA had been determined, cDNA was prepared through reverse transcription. The PCR conditions were the following: predenaturation at $95{ }^{\circ} \mathrm{C}$ for 1 minute; denaturation at $95{ }^{\circ} \mathrm{C}$ for 15 seconds, annealing at $58^{\circ} \mathrm{C}$ for 20 seconds, extension at $72{ }^{\circ} \mathrm{C}$ for 20 seconds, for 40 cycles; then, extension at $72{ }^{\circ} \mathrm{C}$ for 5 minutes to terminate the reaction. After the completion of real-time quantitative PCR (RT-qPCR), the reliability of the melting curve and amplification curve results obtained by PCR was quantitatively analyzed, and the cycle threshold $(\mathrm{Ct})$ was set. The ratios of CCL2, CXCL8, CXCL10, PTGS2 and the internal control, $\beta$-actin, were used to represent their relative expression levels, and the relative mRNA expression intensity of each was calculated. There were three duplicate holes in each group, and the test was repeated 3 times.

\section{Statistical analysis}

SPSS 22.0 (IBM Corp., Armonk, NY, USA) and GraphPad Prism 7 (GraphPad Software, Inc., San Diego, CA, USA) were used for data analysis and graphing. Differences between groups were analyzed using $t$-test or one-way analysis of variance. A P value $<0.05$ indicated a statistically significant difference.

\section{Results}

\section{Retrieval of putative ingredient targets}

Using the keywords and criteria described above, we retrieved 24 active compounds and 360 putative target genes of HQ from the TCMSP database, the names of which were subsequently converted into gene ID using Uniprot. Detailed information of the selected ingredients is displayed in Table 1.

\section{Identification of compound-disease-related genes}

The search of the GeneCards database using the term "colorectal cancer" identified 9,876 CRC-related genes. The compound targets and the disease targets were intersected, and the overlapping genes between HQ and CRC were visualized in a Venn diagram (Figure 1).

\section{Network visualization}

The HQ-CRC network, which was constructed in Cytoscape, comprised 177 nodes and 318 edges. The network is displayed in Figure 2. Sixteen candidate compounds had a median of 13 degrees, which suggested that most HQ compounds affected multiple targets. Quercetin, kaempferol, and formononetin were found to act on 129, 47, and 24 targets, respectively. The OB of quercetin, kaempferol, and formononetin was $46.43 \%$, $41.88 \%$, and $69.67 \%$, respectively, indicating that they might be the active compounds of HQ due to their considerable positioning in the network.

Overlapping genes were input into the STRING database to construct a PPI network with a minimum required connection score of 0.4. As shown in Figure 3A, the PPI network comprised 161 nodes and 2,662 edges, with an average degree of 33.1. Within the network, AKT1, interleukin (IL)-6, and vascular endothelial growth factor A (VEGFA) were the predominant genes, with 109, 101, and 97 interactions, respectively. The top 30 genes with the highest number of interactions are shown in Figure $3 B$.

In order to reveal the mechanisms underlying the effects of HQ on CRC, the PPI network of HQ putative targets and the PPI network of CRC-related targets were merged to identify the candidate targets for HQ against CRC. This network consisted of 5,706 nodes and 139,142 edges, and is presented in Figure 4A. A network of significant targets for HQ against CRC was constructed, and it contained 1,327 nodes and 58,194 edges (Figure 4B). The median values of degree centrality (DC) and BC were 61 and 4,664, respectively. The candidate targets were further screened, and 61 targets with $\mathrm{DC}>61$ and $\mathrm{BC}>4,664$ were identified (Figure 4C). A total of 61 core target genes of HQ in CRC were eventually identified. 
Table 1 The final selected compounds in Astragalus membranaceus for analysis

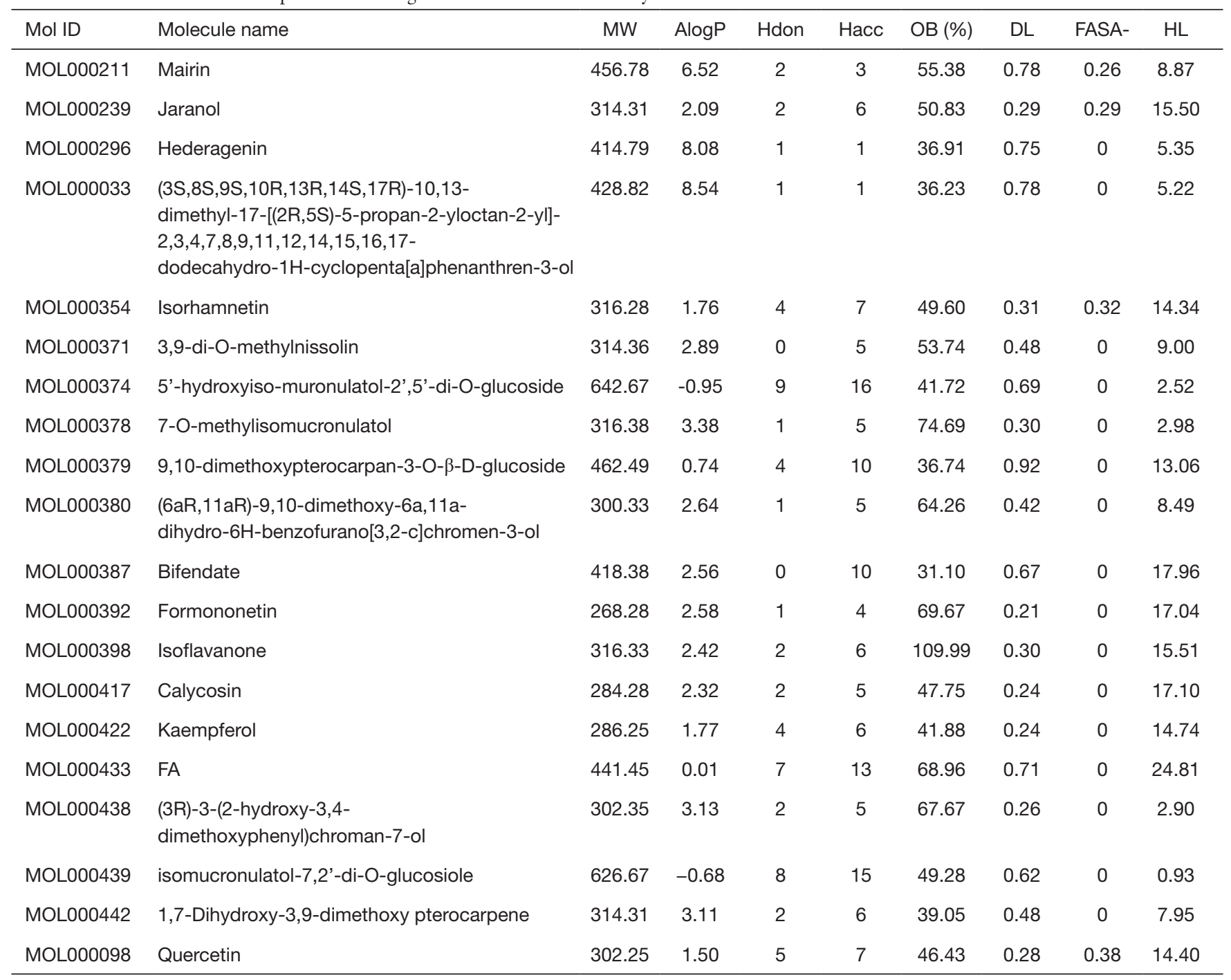

$\mathrm{OB}$, oral bioavailability; DL, drug-likeness; MW, molecular weight; AlogP, lipid-water partition coefficient log P; Hdon, hydrogen-bond donors; Hacc, hydrogen-bond acceptors; FASA-, fractional negative accessible surface area; HL, half-life.

\section{GO and KEGG enrichment analysis}

The names of overlapping genes between HQ and CRC were converted into symbol ID with the Uniprot database serving as a reference. In regard to the GO enrichment analysis, the genes were enriched in response to lipopolysaccharide (LPS), RNA polymerase II transcription factor complex, and DNA-binding transcription activator activity, with an adjusted $\mathrm{P}$ value of $2.10 \mathrm{E}-28,5.98 \mathrm{E}-8$, and $1.77 \mathrm{E}-7$, respectively. Details of the GO enrichment analysis are shown in Table S2, and the top 20 GO terms are shown in Figure 5. The highly enriched GO terms in biological processes, cellular components, and molecular functions included regulation of gene silencing, regulation of gene expression, nucleoplasm, nucleus, protein binding, and ubiquitin protein ligase binding.

The KEGG enrichment analysis revealed that the genes were enriched in AGE-RAGE, IL-17, and tumor necrosis factor (TNF) signaling pathways, with an adjusted $\mathrm{P}$ value of 4.37E-25, 1.42E-19, and 2.10E-19, respectively. Details of the KEGG enrichment analysis are given in Table S3. In Figure 6, the size of the spot represents the count of genes and the color represents the adjusted $\mathrm{P}$ value. 


\section{Gene-pathway network analysis}

The gene-pathway network was constructed based on the significantly enriched pathways and genes that regulate these pathways, and is presented in Figure 7. Topological analysis of 20 pathways and 88 genes with BC was carried out. The squares represent target genes and the $\mathrm{V}$-shapes represent pathways in the network. The network diagram suggested that AKT1 had the highest $\mathrm{BC}$ and was the core target gene. A number of other genes, including MAPK1, RELA, IKBKB, $C H U K$, and $I L-6$, also had high BC, suggesting that they might be key target genes of HQ in CRC.

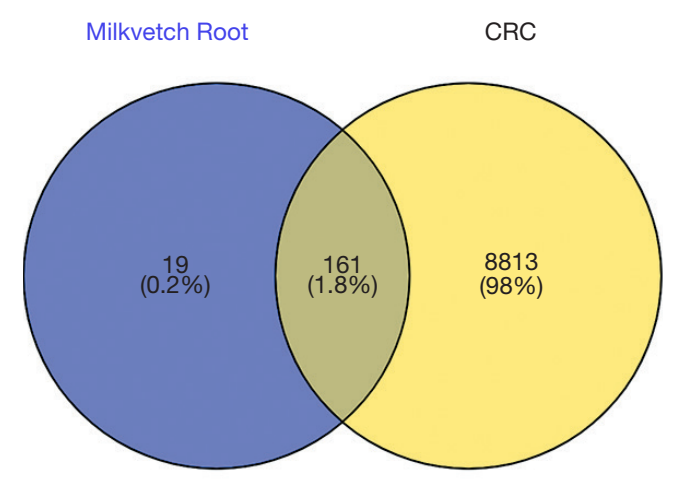

Figure 1 Overlapping targets between potential HQ targets and CRC targets. HQ, Huangqi; CRC, colorectal cancer.

\section{The active component of $H Q$ inhibits the activity of HCT116 cells}

CRC (HCT116) cells were treated with quercetin, isorhamnetin, or formononetin, the three main components of HQ, for 48 hours. The results of CCK- 8 assay showed that compared with the control group, the growth activity of HCT116 cells treated with quercetin, isorhamnetin, or formononetin at a concentration of $20 \mu \mathrm{M}$ was reduced, and this effect was most obvious in the quercetin group (Figure $8 A$ ). The results showed that HQ had an inhibitory effect on the growth of HCT116 cells.

\section{Effect of HQ on IL-17 signaling pathway}

Next, we investigated the mechanism of the inhibitive effect of HQ on HCT116 cells. After 48 hours of drug treatment, compared to that in the control cells, the mRNA expression intensity of CCL2, CXCL8, CXCL10, and PTGS2 was decreased in HCT116 cells in the experimental groups, to varying degrees (Figure 8B).

\section{Discussion}

Previous studies investigating HQ had revealed its antioxidative and anti-inflammatory effects, as well as its enhancement of immune system response, which suggested that HQ could be an alternative potential antitumorigenic agent (26). In addition, formononetin, one of the active

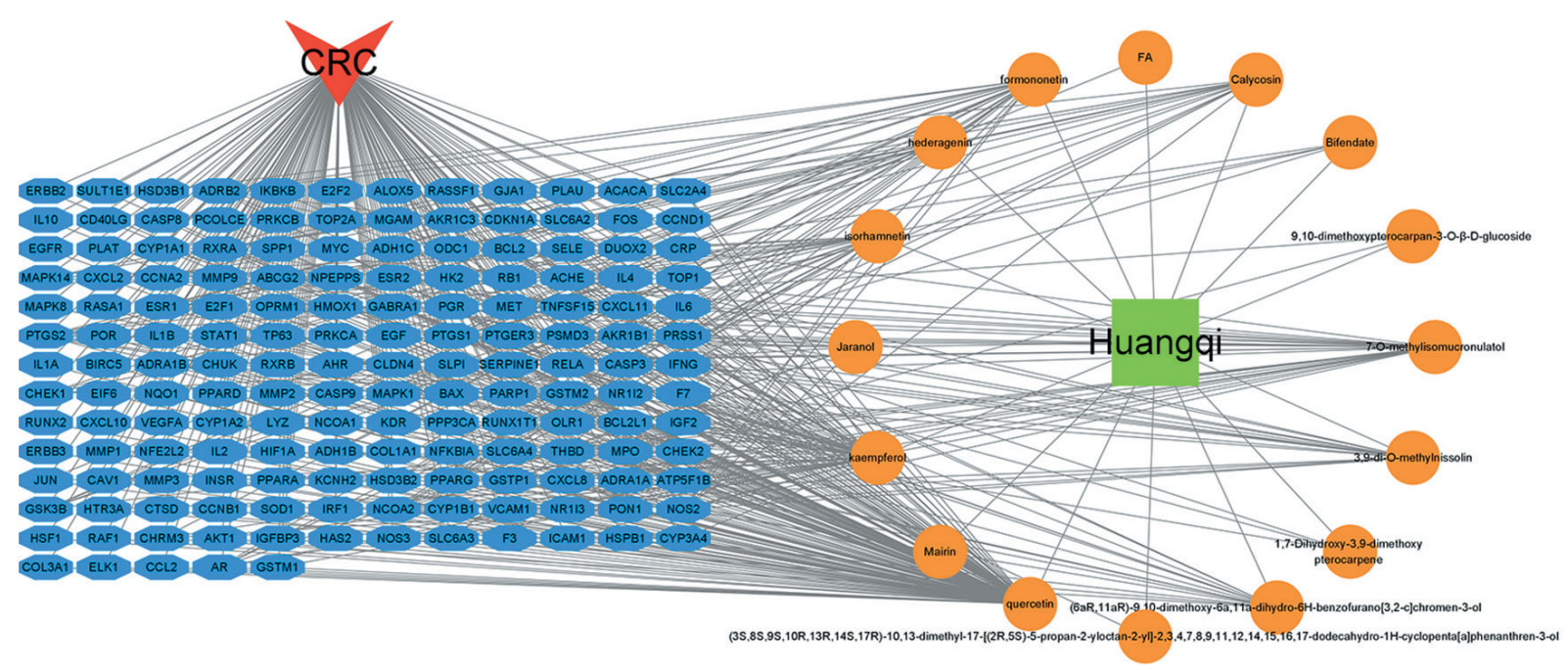

Figure 2 Compound-target network of HQ. The blue triangles represent targets; the orange ones represent the compounds from HQ, respectively. HQ, Huangqi. 


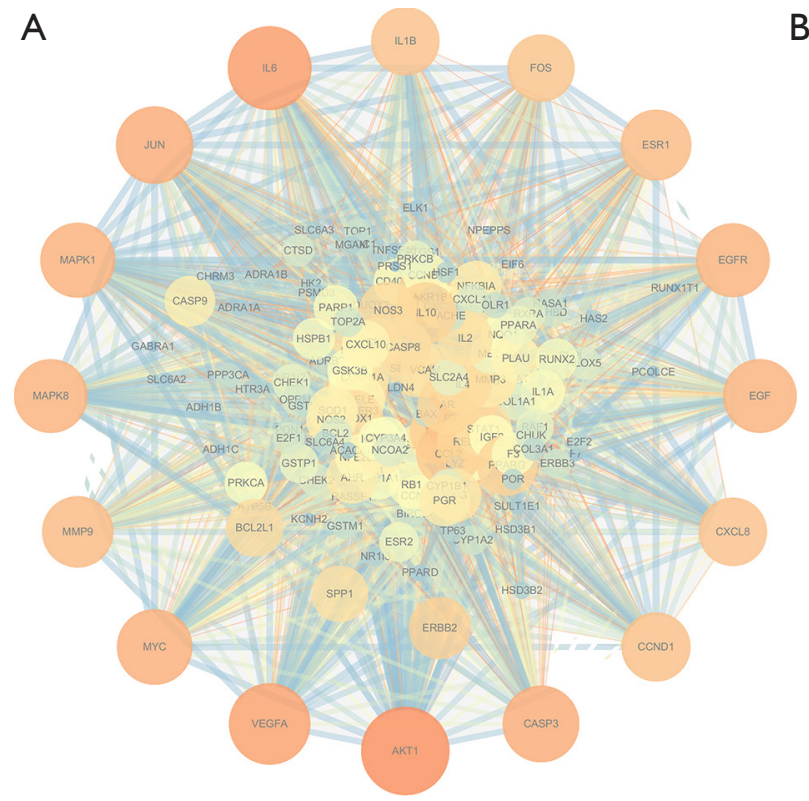

B

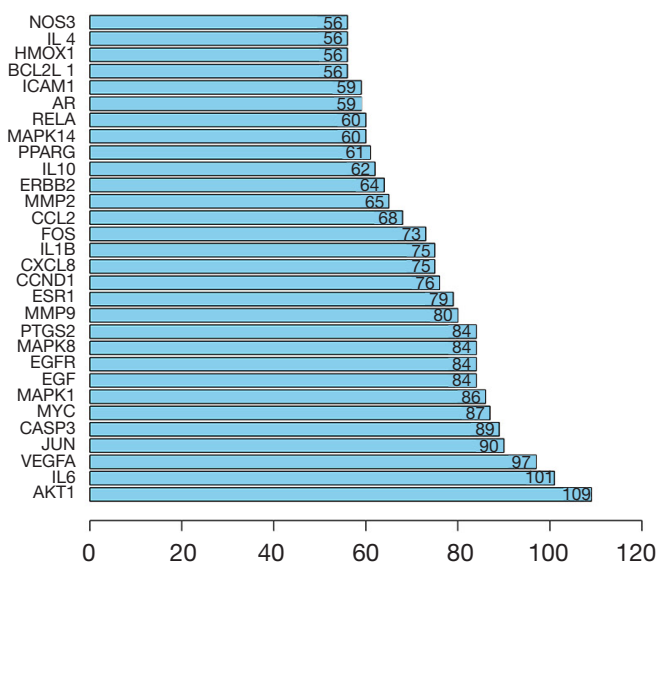

Figure 3 CRC-target STRING network and core genes. (A) Network of the shared targets. The orange nodes represent targets and the node size is proportional to the degree centrality by topology analysis. (B) The top 30 shared targets based on degree centrality. CRC, colorectal cancer.
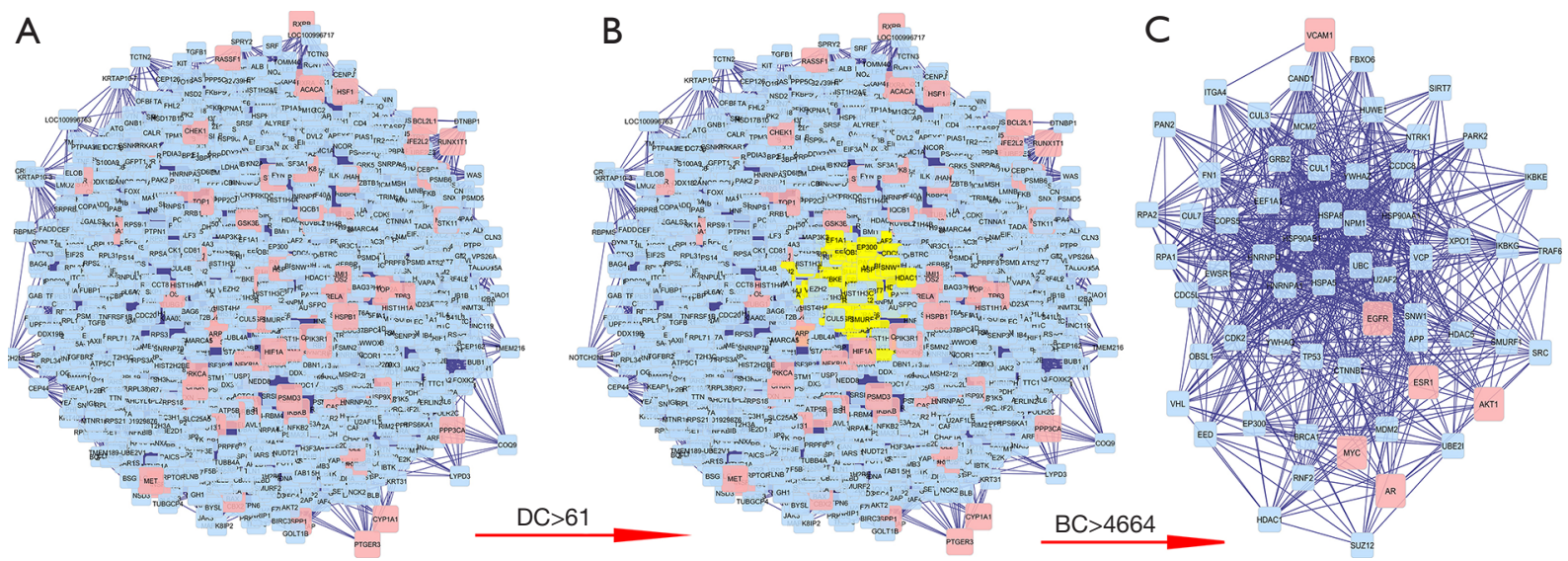

Figure 4 Identification of candidate targets of HQ against CRC. (A) The interactive PPI network of HQ putative targets and CRC-related targets. (B) PPI network of significant proteins extracted from A. (C) PPI network of candidate HQ targets for CRC treatment extracted from B. DC, degree centrality; BC, betweenness centrality; HQ, Huangqi; CRC, colorectal cancer; PPI, protein-protein interaction.

compounds of $\mathrm{HQ}$, was reported to exert an anti-angiogenic effect on colon cancer cells in vitro and in vivo, and in combination with $5-\mathrm{FU}$, it was demonstrated to increase the chemosensitivity of cancer cells, which indicated a synergistic effect with chemotherapy $(15,27)$. However, the specific mechanism of the antitumorigenic effect of HQ at the molecular level requires further investigation.
In enrichment analysis, response to LPS is positioned at a leading place, and LPS is an essential product of Gramnegative microbiota in the gut. In addition, elevated LPS is commonly seen in CRC patients, even those with earlystage adenoma (28). Through its activation of toll-like receptor 4 (TOR4), NF- $\mathrm{NB}$, and $\beta 1$ integrin-mediated cell adhesion, LPS is associated with the progression of CRC 
Biological process
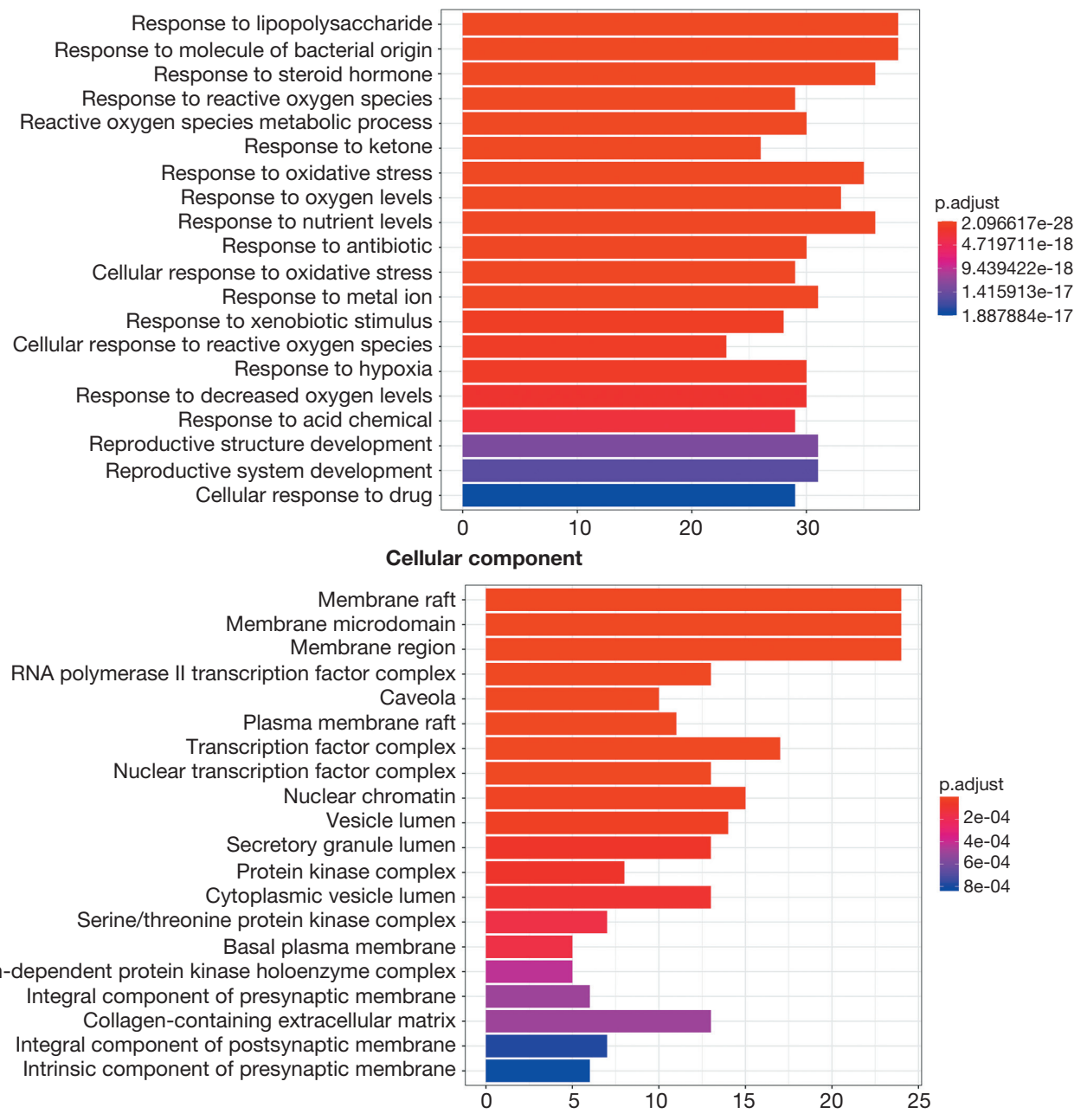

Molecular function

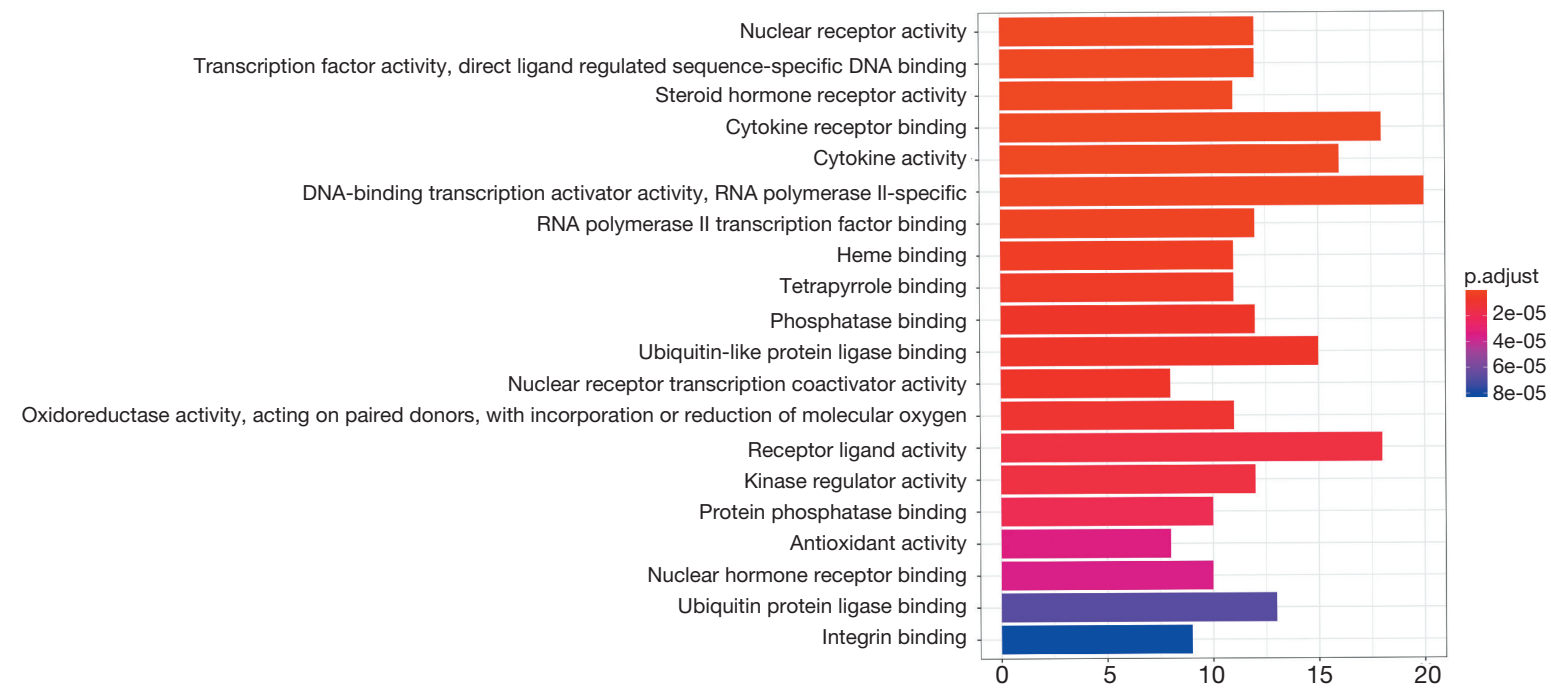

Figure 5 GO terms of candidate targets of HQ against CRC. The top 20 GO functional categories with adjusted P values $<0.05$ were selected. GO, gene ontology; HQ, Huangqi; CRC, colorectal cancer. 


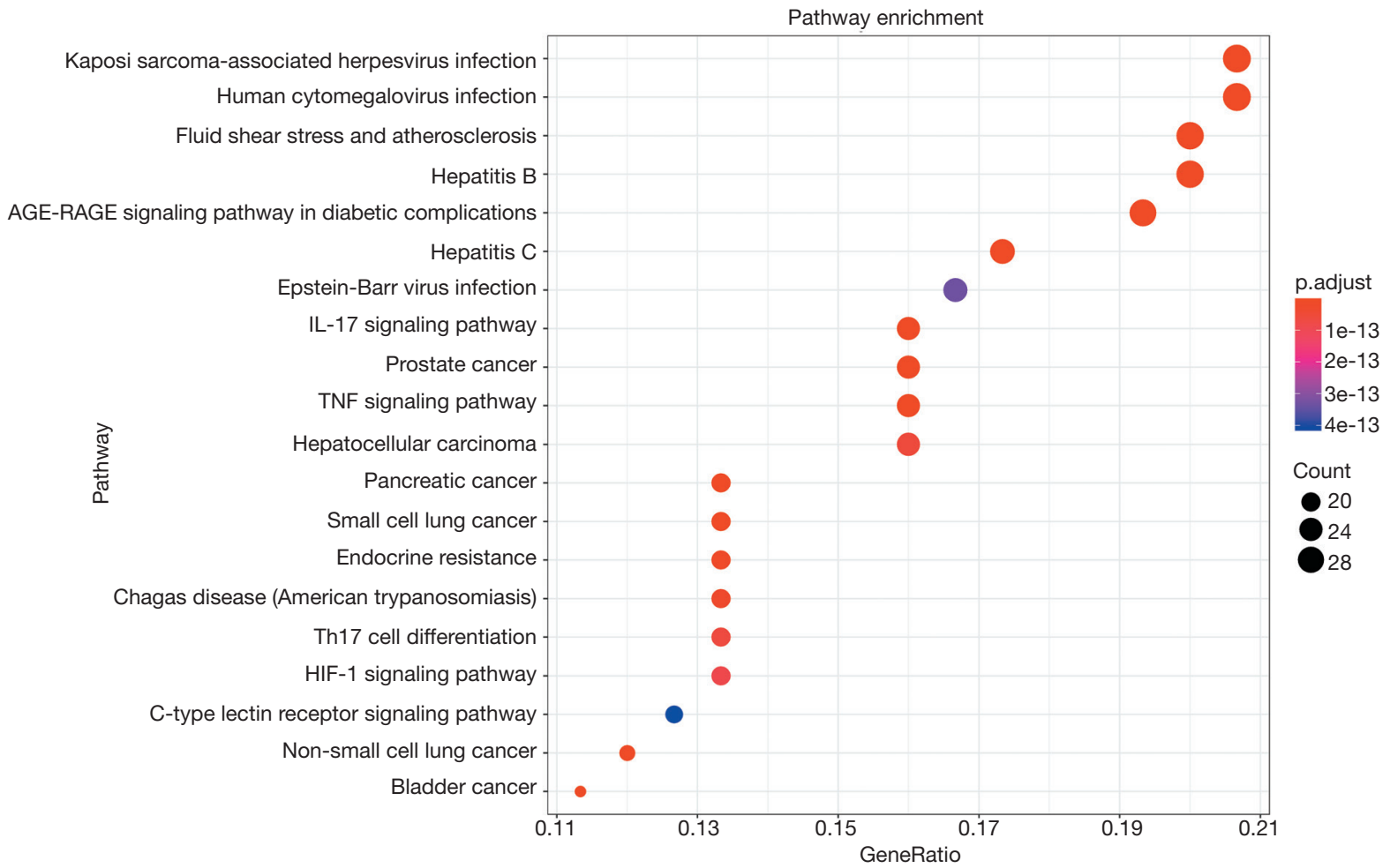

Figure 6 KEGG pathway enrichment of candidate targets of HQ against CRC. Pathways that had significant changes of adjusted P values $<0.05$ were identified. The size of the spot represents the count of genes while the color represents the adjusted P value. KEGG, Kyoto Encyclopedia of Genes and Genomes; HQ, Huangqi; CRC, colorectal cancer.

and promotes the progression of CRC metastasis (29-31), which corroborates the significant role of LPS in CRC treatment. Several studies reported that programmed cell death protein 1/programmed death-ligand 1 (PD-1/PD-L1) blockade therapy was effective in CRC patients and that the efficacy of PD-1-based immunotherapy was affected by the gut microbiome (32-34). Furthermore, a recent study to investigate the association between LPS and the failure of PD-1/PD-L1 therapy concluded that LPS was potentially the main reason for the failure of $\mathrm{PD}-1 / \mathrm{PD}-\mathrm{L} 1$ therapy in CRC patients (35). Combined with the results of this network analysis, HQ was revealed to respond strongly to LPS, which might indicate its capacity to regulate the level of LPS and its potential synergistic effect with CRC immunotherapy. Furthermore, according to a recent study, LAYN expression could be adopted as a prognostic biomarker to determine immune infiltration in CRC (36), which could be a potential method to further validate the effect of HQ.

Moreover, GO analysis also showed response to oxidative stress to be enriched. Some studies have reported that oxidation promotes the progression of CRC $(37,38)$. For example, in colorectal cells, the butyrate level was found to be associated with the butyrate-induced pro-apoptotic process; as a result, oxidation of butyrate affected the butyrate protective effect in CRC. Fatty acid oxidation was also found to lead to CRC progression, and fatty acid oxidation-derived energy can induce tumor metastasis (39). Carnitine palmitoyl transferase 1 (CPT1), a rate-limiting fatty acid oxidation enzyme responsible for transporting fatty acid into mitochondria, has been found to be associated with CRC, and inhibition of CPT1A showed a positive outcome in CRC treatment (37). Together with the results of the present network analysis, these findings suggest that HQ is capable of regulating oxidative process, which might indicate an antitumorigenic effect.

In this study, KEGG enrichment analysis showed that the AGE-RAGE signaling pathway was ranked first, followed by inflammation-related pathways, such as the IL17 and TNF signaling pathways. Excessive endogenous or exogenous advanced glycation end products (AGEs) have been implicated in cancer development (40). Receptor 


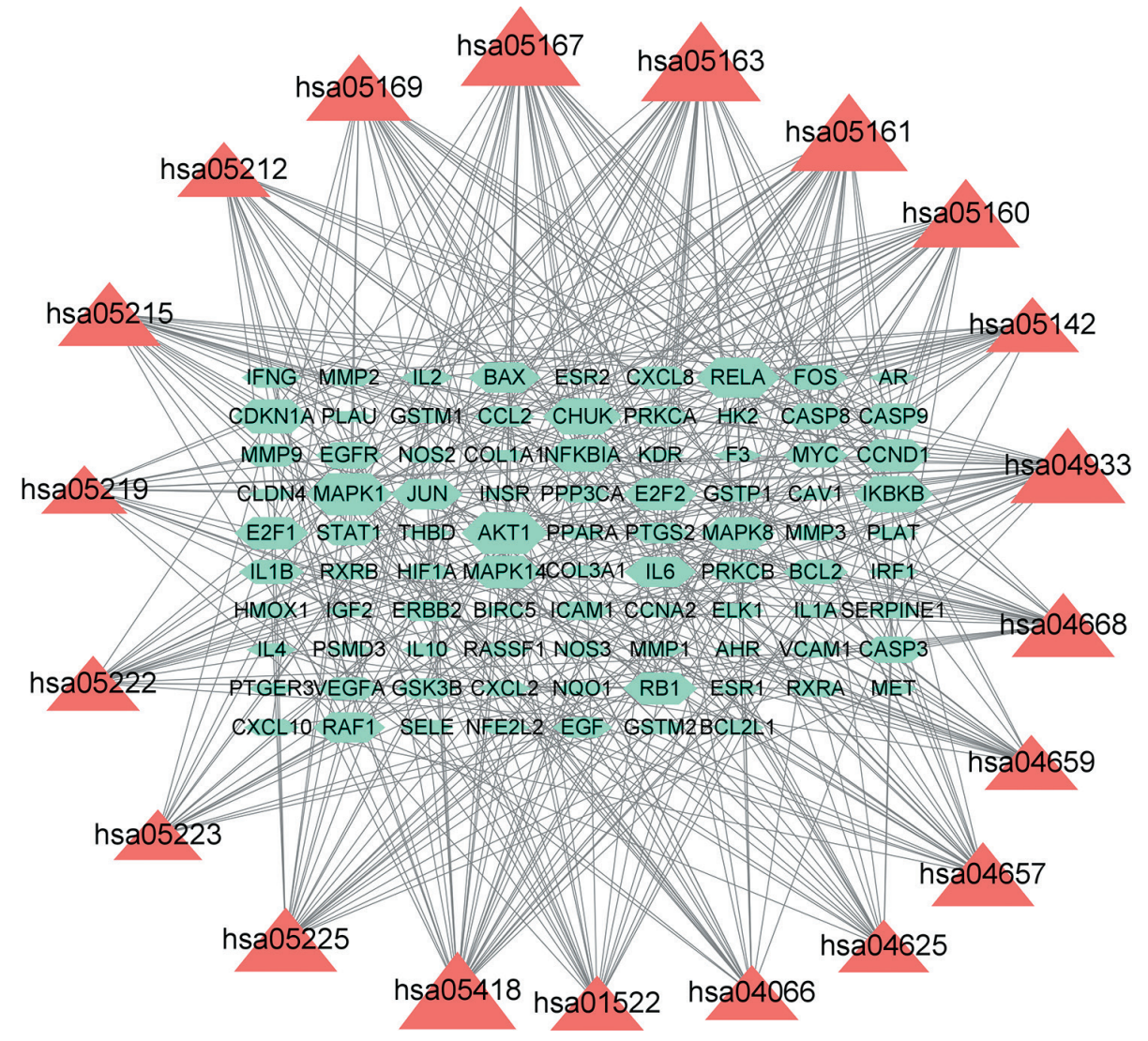

Figure 7 Gene-pathway network of HQ against CRC. Topological analysis of 20 pathways and 88 genes with betweenness centrality was carried out. The cyan squares represent target genes, and the red triangles represent pathways. A larger size represents higher betweenness centrality. HQ, Huangqi; CRC, colorectal cancer.
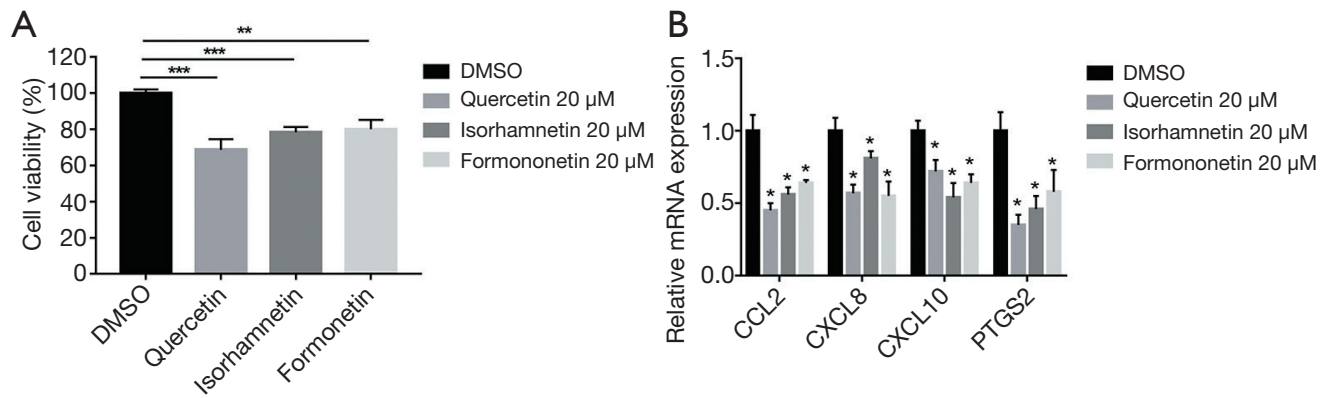

Figure 8 The IL-17 signaling pathway was inhibited in CRC cells and regulated cell proliferation. (A) Cell activity was detected using a Cell Counting Kit-8 assay. (B) The expression of downstream IL-17 signaling pathway genes in drug-treated cells was determined by RTqPCR. Control group: DMSO; experimental groups: quercetin $20 \mu \mathrm{M}$, isorhamnetin $20 \mu \mathrm{M}$, formononetin $20 \mu \mathrm{M}$ ( ${ }^{*}$, $\mathrm{P}<0.05$; **, $\mathrm{P}<0.01$; ***, $\mathrm{P}<0.001)$. IL; interleukin; CRC, colorectal cancer. 
for advanced glycation end products (RAGE) activation initiates downstream signaling pathways to promote inflammation, inhibit apoptosis, increase angiogenesis, and tumor growth and invasion (41). Specifically, the expression of AGE-RAGE was detected in colorectal carcinoma, and even in precancerous lesions such as colon adenomas, and has been validated by in vitro experiments and in vivo animal models (42-44).

Inflammation-related pathways were also enriched in the KEGG analysis. Development from normal cells to CRC requires a number of inflammatory factors, and CRC is more likely to occur in patients with chronic or inflammatory bowel disease (45). The activation of immune signaling pathways by bacteria leads to a loss of hemostasis and creates a proneoplastic inflammatory environment. Both inflammasome activation and NF- $\kappa \mathrm{B}$ pathway were reported to be stimulated by mutational scenario or microbes, and the NF- $\kappa \mathrm{B}$ pathway was shown to mediate the production of cytokines, like IL-6, which is one of the pathogenic factors in CRC (46). The enrichment of two major enriched pathways reported in this study, the AGERAGE and inflammation-related signaling pathways, confirms the antitumorigenic effect of $\mathrm{HQ}$.

Nevertheless, several limitations of this network analysis should be taken into consideration. First, owing to polypharmacy of TCM, the retrieved active compounds were predicted and might not be the actual molecules absorbed. Second, HQ consists of several compounds with different formulations; therefore, the antitumorigenic effects of the individual active compounds are still elusive. Finally, research results need to be validated in a cellular or animal model. Therefore, we performed in vitro experiments, which confirmed that the three active components of HQ, quercetin, isorhamnetin, and formononetin, promote the apoptosis of CRC cells by reducing the expression of CCL2, CXCL8, CXCL10, PTGS2, and other genes. We also identified CCL2, CXCL8, CXCL10, and PTGS2 as key genes in the IL-17 signaling pathway. Together, our findings verified the antitumor activity of $\mathrm{HQ}$.

\section{Conclusions}

The results of this network pharmacology analysis revealed that HQ exerts a potential antitumorigenic effect on CRC by interfering with oxidation and inflammationrelated processes, which may provide a novel approach for combination treatment in CRC patients receiving immunotherapy.

\section{Acknowledgments}

Funding: This research was supported by the Chair Professor Foundation of the First Affiliated Hospital of Jinan University (702023), the Research Incubation Fund (802175), the Guangdong education department (2018GWQNCX050) and the Basic and Applied Basic Research Fund of Guangdong Province (2019A1515011763; 2019A1515110543).

\section{Footnote}

Reporting Checklist: The authors have completed the MDAR reporting checklist. Available at http://dx.doi.org/10.21037/ tcr-20-2596

Conflicts of Interest: All authors have completed the ICMJE uniform disclosure form (available at http://dx.doi. org/10.21037/tcr-20-2596). The authors have no conflicts of interest to declare.

Ethical Statement: The authors are accountable for all aspects of the work in ensuring that questions related to the accuracy or integrity of any part of the work are appropriately investigated and resolved. The study was conducted in accordance with the Declaration of Helsinki (as revised in 2013). This research does not involve human subjects, human materials, and therefore is not subject to approval of an institutional ethics committee.

Open Access Statement: This is an Open Access article distributed in accordance with the Creative Commons Attribution-NonCommercial-NoDerivs 4.0 International License (CC BY-NC-ND 4.0), which permits the noncommercial replication and distribution of the article with the strict proviso that no changes or edits are made and the original work is properly cited (including links to both the formal publication through the relevant DOI and the license). See: https://creativecommons.org/licenses/by-nc-nd/4.0/.

\section{References}

1. Estimated cancer incidence, mortality and prevalence worldwide in 2012. WHO, 2012.

2. Kuipers EJ, Grady WM, Lieberman D, et al. Colorectal cancer. Nat Rev Dis Primers 2015;1:15065.

3. Classics in oncology. Heredity with reference to carcinoma as shown by the study of the cases examined in the 
pathological laboratory of the University of Michigan, 1895-1913. By Aldred Scott Warthin. 1913. CA Cancer J Clin 1985;35:348-59.

4. Cheng L, Eng C, Nieman LZ, et al. Trends in colorectal cancer incidence by anatomic site and disease stage in the United States from 1976 to 2005. Am J Clin Oncol 2011;34:573-80.

5. Papamichael D, Audisio RA, Glimelius B, et al. Treatment of colorectal cancer in older patients: International Society of Geriatric Oncology (SIOG) consensus recommendations 2013. Ann Oncol 2015;26:463-76.

6. Hurwitz H, Fehrenbacher L, Novotny W, et al. Bevacizumab plus irinotecan, fluorouracil, and leucovorin for metastatic colorectal cancer. N Engl J Med 2004;350:2335-42.

7. Heemskerk-Gerritsen BA, Rookus MA, Aalfs CM, et al. Improved overall survival after contralateral risk-reducing mastectomy in BRCA1/2 mutation carriers with a history of unilateral breast cancer: a prospective analysis. Int J Cancer 2015;136:668-77.

8. Tao YG, Huang XF, Wang JY, et al. Exploring Molecular Mechanism of Huangqi in Treating Heart Failure Using Network Pharmacology. Evid Based Complement Alternat Med 2020;2020:6473745.

9. Chen M, May BH, Zhou IW, et al. FOLFOX 4 combined with herbal medicine for advanced colorectal cancer: a systematic review. Phytother Res 2014;28:976-91.

10. Qi F, Zhao L, Zhou A, et al. The advantages of using traditional Chinese medicine as an adjunctive therapy in the whole course of cancer treatment instead of only terminal stage of cancer. Biosci Trends 2015;9:16-34.

11. Tin MM, Cho CH, Chan K, et al. Astragalus saponins induce growth inhibition and apoptosis in human colon cancer cells and tumor xenograft. Carcinogenesis 2007;28:1347-55.

12. Cui R, He J, Wang B, et al. Suppressive effect of Astragalus membranaceus Bunge on chemical hepatocarcinogenesis in rats. Cancer Chemother Pharmacol 2003;51:75-80.

13. Chen Z, Wang X, Li Y, et al. Comparative Network Pharmacology Analysis of Classical TCM Prescriptions for Chronic Liver Disease. Front Pharmacol 2019;10:1353.

14. Law PC, Auyeung KK, Chan LY, et al. Astragalus saponins downregulate vascular endothelial growth factor under cobalt chloride-stimulated hypoxia in colon cancer cells. BMC Complement Altern Med 2012;12:160.

15. Tian QE, De Li H, Yan M, et al. Effects of Astragalus polysaccharides on P-glycoprotein efflux pump function and protein expression in H22 hepatoma cells in vitro.
BMC Complement Altern Med 2012;12:94.

16. Tseng A, Yang CH, Chen $\mathrm{CH}$, et al. An in vivo molecular response analysis of colorectal cancer treated with Astragalus membranaceus extract. Oncol Rep 2016;35:659-68.

17. Wang KH, Wu JR, Zhang D, et al. Comparative efficacy of Chinese herbal injections for treating chronic heart failure: a network meta-analysis. BMC Complement Altern Med 2018;18:41.

18. Chen P, Ni W, Xie T, et al. Meta-Analysis of 5-Fluorouracil-Based Chemotherapy Combined With Traditional Chinese Medicines for Colorectal Cancer Treatment. Integr Cancer Ther 2019;18:1534735419828824.

19. Chen M, May BH, Zhou IW, et al. Meta-Analysis of Oxaliplatin-Based Chemotherapy Combined With Traditional Medicines for Colorectal Cancer: Contributions of Specific Plants to Tumor Response. Integr Cancer Ther 2016;15:40-59.

20. Hopkins AL. Network pharmacology: the next paradigm in drug discovery. Nat Chem Biol 2008;4:682-90.

21. Hopkins AL. Network pharmacology. Nat Biotechnol 2007;25:1110-1.

22. Zhang R, Zhu X, Bai H, et al. Network Pharmacology Databases for Traditional Chinese Medicine: Review and Assessment. Front Pharmacol 2019;10:123.

23. Hao da C, Xiao PG. Network pharmacology: a Rosetta Stone for traditional Chinese medicine. Drug Dev Res 2014;75:299-312.

24. Luo TT, Lu Y, Yan SK, et al. Network Pharmacology in Research of Chinese Medicine Formula: Methodology, Application and Prospective. Chin J Integr Med 2020;26:72-80.

25. Zhang W, Huai Y, Miao Z, et al. Systems Pharmacology for Investigation of the Mechanisms of Action of Traditional Chinese Medicine in Drug Discovery. Front Pharmacol 2019;10:743.

26. Ren S, Zhang H, Mu Y, et al. Pharmacological effects of Astragaloside IV: a literature review. J Tradit Chin Med 2013;33:413-6.

27. Auyeung KK, Law PC, Ko JK. Novel anti-angiogenic effects of formononetin in human colon cancer cells and tumor xenograft. Oncol Rep 2012;28:2188-94.

28. Kang M, Edmundson P, Araujo-Perez F, et al. Association of plasma endotoxin, inflammatory cytokines and risk of colorectal adenomas. BMC Cancer 2013;13:91.

29. Killeen SD, Wang JH, Andrews EJ, et al. Bacterial endotoxin enhances colorectal cancer cell adhesion and 
invasion through TLR-4 and NF-kappaB-dependent activation of the urokinase plasminogen activator system. Br J Cancer 2009;100:1589-602.

30. Fukata M, Chen A, Vamadevan AS, et al. Toll-like receptor-4 promotes the development of colitis-associated colorectal tumors. Gastroenterology 2007;133:1869-81.

31. Hsu RY, Chan CH, Spicer JD, et al. LPS-induced TLR4 signaling in human colorectal cancer cells increases beta1 integrin-mediated cell adhesion and liver metastasis. Cancer Res 2011;71:1989-98.

32. Le DT, Uram JN, Wang H, et al. PD-1 Blockade in Tumors with Mismatch-Repair Deficiency. N Engl J Med 2015;372:2509-20.

33. Gopalakrishnan V, Spencer CN, Nezi L, et al. Gut microbiome modulates response to anti-PD-1 immunotherapy in melanoma patients. Science 2018;359:97-103.

34. Matson V, Fessler J, Bao R, et al. The commensal microbiome is associated with anti-PD-1 efficacy in metastatic melanoma patients. Science 2018;359:104-8.

35. Song W, Tiruthani K, Wang Y, et al. Trapping of Lipopolysaccharide to Promote Immunotherapy against Colorectal Cancer and Attenuate Liver Metastasis. Adv Mater 2018;30:e1805007.

36. Pan JH, Zhou H, Cooper L, et al. LAYN Is a Prognostic Biomarker and Correlated With Immune Infiltrates in Gastric and Colon Cancers. Front Immunol 2019;10:6.

37. Wang YN, Zeng ZL, Lu J, et al. CPT1A-mediated fatty acid oxidation promotes colorectal cancer cell metastasis by inhibiting anoikis. Oncogene 2018;37:6025-40.

38. Han A, Bennett N, MacDonald A, et al. Cellular

Cite this article as: Chu $\mathrm{XD}$, Zhang YR, Lin ZB, Zhao Z, Huangfu SC, Qiu SH, Guo YG, Ding H, Huang T, Chu XL, Pan JH, Pan YL. A network pharmacology approach for investigating the multi-target mechanisms of Huangqi in the treatment of colorectal cancer. Transl Cancer Res 2021;10(2):681693. doi: $10.21037 / \mathrm{tcr}-20-2596$
Metabolism and Dose Reveal Carnitine-Dependent and -Independent Mechanisms of Butyrate Oxidation in Colorectal Cancer Cells. J Cell Physiol 2016;231:1804-13.

39. Nieman KM, Kenny HA, Penicka CV, et al. Adipocytes promote ovarian cancer metastasis and provide energy for rapid tumor growth. Nat Med 2011;17:1498-503.

40. Sakellariou S, Fragkou P, Levidou G, et al. Clinical significance of AGE-RAGE axis in colorectal cancer: associations with glyoxalase-I, adiponectin receptor expression and prognosis. BMC Cancer 2016;16:174.

41. Rojas A, Figueroa H, Morales E. Fueling inflammation at tumor microenvironment: the role of multiligand/RAGE axis. Carcinogenesis 2010;31:334-41.

42. Marinakis E, Bagkos G, Piperi C, et al. Critical role of RAGE in lung physiology and tumorigenesis: a potential target of therapeutic intervention? Clin Chem Lab Med 2014;52:189-200.

43. Sasahira T, Akama Y, Fujii K, et al. Expression of receptor for advanced glycation end products and HMGB1/ amphoterin in colorectal adenomas. Virchows Arch 2005;446:411-5.

44. Kuniyasu H, Chihara Y, Kondo H. Differential effects between amphoterin and advanced glycation end products on colon cancer cells. Int J Cancer 2003;104:722-7.

45. Brennan CA, Garrett WS. Gut Microbiota, Inflammation, and Colorectal Cancer. Annu Rev Microbiol 2016;70:395-411.

46. Karin M, Greten FR. NF-kappaB: linking inflammation and immunity to cancer development and progression. Nat Rev Immunol 2005;5:749-59. 
Supplementary

Table S1 Primer sequences

\begin{tabular}{lccc}
\hline Gene name & \multicolumn{1}{c}{ Forward } & Reverse & Amplicon size \\
\hline CCL2 & CAGCCAGATGCAATCAATGCC & TGGAATCCTGAACCCACTTCT & 190 \\
CXCL8 $($ IL8) & TITTGCCAAGGAGTGCTAAAGA & AACCCTCTGCACCCAGTTTC & 194 \\
CXCL10 & GTGGCATTCAAGGAGTACCTC & TGATGGCCTTCGATTCTGGATT & 198 \\
PTGS2 & CTGGCGCTCAGCCATACAG & CGCACTTATACTGGTCAAATCCC & 94 \\
$\beta$-actin & CTCCATCCTGGCCTCGCTGT & GCTGTCACCTTCACCGTTCC & 268 \\
\hline
\end{tabular}




\begin{tabular}{|c|c|c|c|c|c|c|}
\hline Type & ID & Description & $P$ value & $P$ adjust & Gene ID & Count \\
\hline \multirow[t]{20}{*}{$\begin{array}{l}\text { Biological } \\
\text { process }\end{array}$} & GO:0032496 & Response to lipopolysaccharide & $5.10 \mathrm{E}-32$ & $2.10 \mathrm{E}-28$ & $\begin{array}{l}\text { NOS2/PTGS2/MAPK14/RELA/OPRM1/JUN/AKT1/CASP3/MAPK8/CYP1A2/CYP1A1//CAM1/SELE/VCAM1/GSTP1/SLPI/FOS/CASPP/MAPK1/L10//L6/ } \\
\text { NFKBIA/CASP8/PRKCA/GJA1/L1B/CCL2/CXCL8/NOS3/THBD//ERPINE1/MPO/CXCL11/CXCL2/PPARD/HSF1/CXCL10/CHUK }\end{array}$ & 38 \\
\hline & GO:0002237 & Response to molecule of bacterial origin & $2.22 \mathrm{E}-31$ & 4.56E-28 & $\begin{array}{l}\text { NOS2/PTGS2/MAPK14/RELA/OPRM1//UUN/AKT1/CASP3/MAPK8/CYP1A2/CYP1A1//CAM1/SELE/VCAM1/GSTP1/SLPI/FOS/CASPP/MAPK1//L10//L6/ } \\
\text { NFKBIA/CASP8/PRKCA/GJA1/L1B/CCL2/CXCL8/NOS3/THBD/SERPINE1/MPO/CXCL11/CXCL2/PPARD/HSF1/CXCL10/CHUK }\end{array}$ & 38 \\
\hline & GO:0048545 & Response to steroid hormone & 4.27E-27 & $5.86 \mathrm{E}-24$ & $\begin{array}{l}\text { PGR/AR/PTGS2/ESR2/NCOA2/RXRA/ESR1/PPARG/NCOA1/RELAARXRB/HSD3B2/HSD3B1/BCL2/CASP3//CAM1/NR1/2/GSTP1/NR1/3/AKR1C3/ } \\
\text { EGFR/CCND1/FOS/CDKN1A/CASPI/L10/RB1/L6/TP63/CAV1/COL1A1/PARP1/CLDN4/PPARA/PPARD/SPP1 }\end{array}$ & 36 \\
\hline & GO:0000302 & Response to reactive oxygen species & 1.69E-25 & $1.74 \mathrm{E}-22$ & $\begin{array}{l}\text { CCNA2/RELA/MET/JUN/AKT1/BCL2/CASP3/MAPK8/STAT1/HMOX1/CYP1B1/GSTP1/AKR1C3/MMP3/EGFR/FOS/MMP2/MMP9/MAPK1//L10/L6/ } \\
\text { SOD1/NOS3/COLAA1/MPO/NFE2L2/NQO1/HSF//CHUK }\end{array}$ & 29 \\
\hline & GO:0072593 & Reactive oxygen species metabolic process & $3.61 \mathrm{E}-24$ & $2.97 \mathrm{E}-21$ & $\begin{array}{l}\text { NOS2/PTGS2/MAPK14/AKT1/BCL2/CYP1A2/CYP1A1//CAM1/CYP1B1/GSTP1/INSR/AKR1C3/MMP3/EGFR/CDKN1A/EIFG/L10/POR/SOD1/HIIF1A/ } \\
\text { CAV1/L1B/DUOX2/NOS3/IFNG/MPO/NFE2L2/NQO1/CRP/HK2 }\end{array}$ & 30 \\
\hline & GO:1901654 & Response to ketone & $8.95 \mathrm{E}-24$ & $6.14 \mathrm{E}-21$ & $\begin{array}{l}\text { AR/NCOA2/PPARG/AKR1B1/NCOA1/F7/RELA/HSD3B2/HSD3B1/AKT1//CAM1/AHR/AKR1C3/EGFR/CCND1/BCL2L1/FOS/CDKN1A/CASP9/ELK1/ } \\
\text { ACACA/CAV1/PARP1/CLDN4/HSF1/SPP1 }\end{array}$ & 26 \\
\hline & GO:0006979 & Response to oxidative stress & $1.32 \mathrm{E}-23$ & $7.74 \mathrm{E}-21$ & $\begin{array}{l}\text { PTGS1/PTGS2/CCNA2/RELA/MET/JUN/AKT1/BCL2/CASP3/MAPK8/STAT1/HMOX1//CYP1B1/GSTP1/AKR1C3/MMP3/EGFR/FOS/MMP2/MMP9/ } \\
\text { MAPK1//L10//L6/SOD1/HIF1A/DUOX2/NOS3/HSPB1/COL1A1/MPO/NFE2L2/NQ1/PARP1/HSF1/CHUK }\end{array}$ & 35 \\
\hline & GO:0070482 & Response to oxygen levels & $2.60 \mathrm{E}-23$ & $1.34 \mathrm{E}-20$ & $\begin{array}{l}\text { NOS2/PTGS2/PPARG/CCNA2//F7/SLCGA4/AKT1/BCL2/CASP3/HMOX1/CYP1A1//CAM1/NCAM1/PSMD3/SLC2A4/VEGFA/CDKN1A/PLAU/MMP2/ } \\
\text { RAF1/HIF1A/CAV1/MYC/CCNB1/PLAT/COL1A1/NFE2L2/PPARA/PPARD/HSF1/E2F1/NPEPS//HK2 }\end{array}$ & 33 \\
\hline & GO:0031667 & Response to nutrient levels & $3.36 \mathrm{E}-23$ & $1.54 \mathrm{E}-20$ & $\begin{array}{l}\text { PTGS2/PPARG/NCOA1/FT/RELA/ADRB2/OPRM1/SLC6A4/JUN/AKT1/BCL2/MAPK8/STAT1/HMOX1/CYP1A1//CAM1/VCAM1/GSTP1/AKR1C3/EGFR/ } \\
\text { CCND1/CDKN1A/MAPK1/POR/SOD1/L1B/COL1A1/MPO/NFE2L2/NQO1/PPARA/PPARD/HSF1/CXCL10/SPP1/PON1 }\end{array}$ & 36 \\
\hline & GO:0046677 & Response to antibiotic & $2.28 \mathrm{E}-22$ & $9.40 \mathrm{E}-20$ & $\begin{array}{l}\text { RELA/HTR3A/OPRM1/SLC6A3/MET/JUN/BCL2/CASP3/STAT1/HMOX1/CYP1A1//CAM1/VCAM1/CYP1B1/GSTP1/AHR/SLC2A4/CCND1/BCL2L1/ } \\
\text { CASP9//L10/L6/CASP8/SOD1/L2/COL1A1/NFE2L2/NQO1/HSF1/CHUK }\end{array}$ & 30 \\
\hline & GO:0034599 & Cellular response to oxidative stress & $3.38 \mathrm{E}-22$ & 1.27E-19 & $\begin{array}{l}\text { CCNA2/RELA/MET/JUN/AKT1/BCL2/MAPK8/HMOX1/CYP1B1/GSTP1/AKR1C3/MMP3/EGFR/FOS/MMP2/MMP9/MAPK1/L10//L6/SOD1/HIF1A/ } \\
\text { NOS3/HSPB1/MPO/NFE2L2/NQO1/PARP1/HSF1/CHUK }\end{array}$ & 29 \\
\hline & GO:0010038 & Response to metal ion & $3.94 \mathrm{E}-22$ & $1.35 \mathrm{E}-19$ & $\begin{array}{l}\text { PTGS2/SLC6A3/JUN/AKT1/BCL2/CASP3/MAPK8/HMOX1/CYP1A2/CYP1A1//CAM1/VCAM1/PPP3CA/AKR1C3/EGFR/CCND1/FOS/CASP9/MMP9/ } \\
\text { MAPK1/CASPB/SOD1/HIF1A/CAV1/CCNB1/L1A/NFE2L2/NQO1/PARP1/HSF1/CHUK }\end{array}$ & 31 \\
\hline & GO:0009410 & Response to xenobiotic stimulus & $1.97 \mathrm{E}-21$ & $6.22 \mathrm{E}-19$ & $\begin{array}{l}\text { PTGS1/PPARG/CCNA2/FF/RELA/HTR3A/OPRM1/SLC6A3/CYP3A4/CYP1A2/CYP1A1//CAM1/NR1/2/CYP1B1/GSTP1/AHR/PPP3CA/GSTM1/GSTM2/ } \\
\text { EGFR/CASPG/RB1/POR/SOD1/NQO1/CHEK2/HSF1/E2F1 }\end{array}$ & 28 \\
\hline & GO:0034614 & Cellular response to reactive oxygen species & $2.78 \mathrm{E}-21$ & $8.18 \mathrm{E}-19$ & $\begin{array}{l}\text { CCNA2/RELA/MET/UUN/AKT1/MAPK8/CYP1B1/AKR1C3/MMP3/EGFR/FOS/MMP2/MMP9/MAPK1/L10/L6/SOD1/NOS3/MPO/NFE2L2/NQO1/HSF1/ } \\
\text { CHUK }\end{array}$ & 23 \\
\hline & GO:0001666 & Response to hypoxia & $3.42 \mathrm{E}-21$ & $9.37 \mathrm{E}-19$ & $\begin{array}{l}\text { NOS2/PTGS2/CCNA2/F7/SLC6A4/AKT1/BCL2/CASP3/HMOX1/CYP1A1//CAM1/VCAM1/PSMD3/SLC2A4/VEGFA/PLAU/MMP2/RAF1/HIF1A/CAV1/ } \\
\text { MYC/CCNB1/PLAT/NFE2L2/PPARA/PPARD/HSF1/E2F1/NPEPS//HK2 }\end{array}$ & 30 \\
\hline & GO:0036293 & Response to decreased oxygen levels & $8.14 \mathrm{E}-21$ & $2.09 \mathrm{E}-18$ & $\begin{array}{l}\text { NOS2/PTGS2/CCNA2/F7/SLCGA4/AKT1/BCL2/CASP3/HMOX1/CYP1A1//CAM1/VCAM1/PSMD3/SLC2A4/VEGFA/PLAU/MMP2/RAF1/HIF1A/CAV1/ } \\
\text { MYC/CCNB1/PLAT/NFE2L2/PPARA/PPARD/HSF1/E2F/NPEPPS/HK2 }\end{array}$ & 30 \\
\hline & GO:0001101 & Response to acid chemical & $1.21 \mathrm{E}-20$ & $2.93 \mathrm{E}-18$ & $\begin{array}{l}\text { PTGS2/RXRA/PPARG/AKR1B1/NCOA1//F7/RELA/SLC6A4/RXRB/KDR/AKT1/CASP3//CAM1/GSTP1/AKR1C3/EGFR/VEGFA/BCL2L1/MMP2/ACACA/ } \\
\text { GJA1/CCNB1/COL1A1/NQ01/COL3A1/HSF//CHUK/E2F1/PON1 }\end{array}$ & 29 \\
\hline & GO:0048608 & Reproductive structure development & $5.76 \mathrm{E}-20$ & $1.32 \mathrm{E}-17$ & $\begin{array}{l}\text { PGR/AR/PTGS2/RXRA/ESR1/PPARG/MAPK14/NCOA1/AKT1/BCL2/BAX/CASP3//CAM1/INSR/AKR1C3/EGFR/NEGFA/CCND1/BCL2L1/MAPK1//L10/ } \\
\text { TP63/CASP8/SOD1//IIF1A/GJA1/NOS3/PPARD/HSF1/SPP1//GF2 }\end{array}$ & 31 \\
\hline & GO:0061458 & Reproductive system development & $7.05 \mathrm{E}-20$ & 1.53E-17 & $\begin{array}{l}\text { PGR/AR/PTGS2/RXRA/ESR1/PPARG/MAPK14/NCOA1/AKT1/BCL2/BAX/CASP3//CAM1/INSR/AKR1C3/EGFR/VEGFA/CCND1/BCL2L1/MAPK1//L10/ } \\
\text { TP63/CASP8/SOD1//IIF1A/GJA1/NOS3/PPARD/HSF1/SPP1/GF2 }\end{array}$ & 31 \\
\hline & GO:0035690 & Cellular response to drug & $9.18 \mathrm{E}-20$ & 1.89E-17 & $\begin{array}{l}\text { NOS2/PTGS2/CHRM3/CCNA2/NCOA1/RELA/OPRM1//KCNH2/KDR/MET/HMOX1//CAM1/CYP1B1/AHR/PPP3CA/GSTM2/MMP3/EGFR/CASP9/ } \\
\text { MAPK1/L10/L6/SOD1/MYC/L1B/NFE2L2/NQO1//CHEK2/HSF1 }\end{array}$ & 29 \\
\hline \multirow[t]{20}{*}{$\begin{array}{l}\text { Cellular } \\
\text { component }\end{array}$} & GO:0045121 & Membrane raft & $1.08 \mathrm{E}-16$ & $1.74 \mathrm{E}-14$ & $\begin{array}{l}\text { PTGS2/ADRA1B/SLC6A2/OLR1/OPRM1/ADRA1A/SLC6A3/SLC6A4/KDR/KKKB/CASP3/HMOX1//CAM1/SELE/HAS2/SLC2A4/INSR/EGFR/MAPK1/ } \\
\text { CASP8/CAV1/GJA1/NOS3/CTSD }\end{array}$ & 24 \\
\hline & GO:0098857 & Membrane microdomain & 1.17E-16 & $1.74 \mathrm{E}-14$ & $\begin{array}{l}\text { PTGS2/ADRA1B/SLC6A2/OLR1/OPRM1/ADRA1A/SLC6A3/SLC6A4/KDR/KBKB/CASP3/HMOX1//CAM1/SELE/HAS2/SLC2A4/INSR/EGFR/MAPK1/ } \\
\text { CASP8/CAV1/GJA1/NOS3/CTSD }\end{array}$ & 24 \\
\hline & GO:0098589 & Membrane region & $2.72 \mathrm{E}-16$ & $2.70 \mathrm{E}-14$ & $\begin{array}{l}\text { PTGS2/ADRA1B/SLC6A2/OLR1/OPRM1/ADRA1A/SLC6A3/SLC6A4/KDR/IKBKB/CASP3/HMOX1//CAM1/SELE/HAS2/SLC2A4/INSR/EGFR/MAPK1/ } \\
\text { CASP8/CAV1/GJA1/NOS3/CTSD }\end{array}$ & 24 \\
\hline & GO:0090575 & RNA polymerase II transcription factor complex & 8.63E-10 & $5.98 \mathrm{E}-08$ & RXRA/PPARG/RXRB/JUN/NR1/2/NR1/3/FOS/RB1/HIF1A/PPARA/PPARD/E2F1/E2F2 & 13 \\
\hline & GO:0005901 & Caveola & $1.00 \mathrm{E}-09$ & $5.98 \mathrm{E}-08$ & PTGS2/ADRA1B/ADRA1A/SLC6A3/HMOX1/SELE/INSR/MAPK1/CAV1/NOS3 & 10 \\
\hline & GO:0044853 & Plasma membrane raft & 1.47E-09 & 7.30E-08 & PTGS2/ADRA1B/ADRA1A/SLC6A3/HMOX1/SELE/HAS2/INSR/MAPK1/CAV1/NOS3 & 11 \\
\hline & GO:0005667 & Transcription factor complex & $8.29 \mathrm{E}-09$ & 3.53E-07 & RXRA/PPARG/RELA/RXRB/JUN/NR1/2/AHR/NR1/3/FOS/RB1/HIF1A/PARP1/PPARA/PPARD/RUNX2/E2F1/E2F2 & 17 \\
\hline & GO:0044798 & Nuclear transcription factor complex & $1.11 \mathrm{E}-08$ & 4.13E-07 & RXRA/PPARG/RXRB/JUN/NR1/2/NR1/3/FOS/RB1/HIF1A/PPARA/PPARD/E2F1/E2F2 & 13 \\
\hline & GO:0000790 & Nuclear chromatin & 4.97E-07 & $1.65 \mathrm{E}-05$ & AR/RXRA/ESR1/NCOA1/RELA/JUN/STAT1/RB1/TP63/HIF1A/MYC/PPARD/RUNX2/E2F1/IRF1 & 15 \\
\hline & GO:0031983 & Vesicle lumen & $7.82 \mathrm{E}-07$ & $2.33 \mathrm{E}-05$ & LYZ/MAPK14/ALOX5/GSTP1/PSMD3/SLPI/EGFR/VEGFA/MAPK1/EGF/SERPINE1/MPO/CTSD//GF2 & 14 \\
\hline & GO:0034774 & Secretory granule lumen & 2.44E-06 & $6.61 \mathrm{E}-05$ & LYZ/MAPK14/ALOX5/GSTP1/PSMD3/SLPI/VEGFA/MAPK1/EGF/SERPINE1/MPO/CTSD/IGF2 & 13 \\
\hline & GO:1902911 & Protein kinase complex & $3.14 \mathrm{E}-06$ & $7.79 E-05$ & CCNA2/IKBKB/INSR/CCND1/CDKN1A/RB1/CCNB1/CHUK & 8 \\
\hline & GO:0060205 & Cytoplasmic vesicle lumen & 4.29E-06 & $9.84 \mathrm{E}-05$ & LYZ/MAPK14/ALOX5/GSTP1/PSMD3/SLPI/VEGFA/MAPK1/EGF/SERPINE1/MPO/CTSD/IGF2 & 13 \\
\hline & GO:1902554 & Serine/threonine protein kinase complex & $7.74 \mathrm{E}-06$ & 0.000156 & CCNA2//KBKB/CCND1/CDKN1A/RB1/CCNB1/CHUK & 7 \\
\hline & GO:0009925 & Basal plasma membrane & $7.84 \mathrm{E}-06$ & 0.000156 & MET/EGFR/ERBB2/CLDN4/ERBB3 & 5 \\
\hline & GO:0000307 & $\begin{array}{l}\text { Cyclin-dependent protein kinase holoenzyme } \\
\text { complex }\end{array}$ & 2.27E-05 & 0.000423 & CCNA2/CCND1/CDKN1A/RB1/CCNB1 & 5 \\
\hline & GO:0099056 & Integral component of presynaptic membrane & 2.93E-05 & 0.000497 & CHRM3/HTR3A/OPRM1/ADRA1A/SLCGA3/SLC6A4 & 6 \\
\hline & GO:0062023 & Collagen-containing extracellular matrix & $3.00 E-05$ & 0.000497 & PRSS1/F7/ACHE/ICAM1/SLPI/MMP2/MMP9//F3/SERPINE1/COL1A1/COL3A1/CTSD/PCOLCE & 13 \\
\hline & GO:0099055 & Integral component of postsynaptic membrane & 4.96E-05 & 0.000779 & CHRM3/GABRA1/HTR3A/OPRM1/ADRA1A/SLC6A3/SLC6A4 & 7 \\
\hline & GO:0098889 & Intrinsic component of presynaptic membrane & $5.67 \mathrm{E}-05$ & 0.00084 & CHRM3/HTR3A/OPRM1/ADRA1A/SLC6A3/SLC6A4 & 6 \\
\hline \multirow{20}{*}{$\begin{array}{l}\text { Molecular } \\
\text { function }\end{array}$} & GO:0004879 & Nuclear receptor activity & $8.43 \mathrm{E}-15$ & $2.10 \mathrm{E}-12$ & PGR/AR/ESR2/RXRA/ESR1/PPARG/RXRB/NR1/2/AHR/NR11//PPARA/PPARD & 12 \\
\hline & GO:0098531 & $\begin{array}{l}\text { Transcription factor activity, direct ligand } \\
\text { regulated sequence-specific DNA binding }\end{array}$ & $8.43 \mathrm{E}-15$ & $2.10 \mathrm{E}-12$ & PGR/AR/ESR2/RXRA/ESR1/PPARG/RXRB/NR1/2/AHR/NR1/3/PPARA/PPARD & 12 \\
\hline & GO:0003707 & Steroid hormone receptor activity & $2.62 \mathrm{E}-12$ & $4.35 \mathrm{E}-10$ & PGR/AR/ESR2/RXRA/ESR1/PPARG/RXRB/NR1/2/NR1/3/PPARA/PPARD & 11 \\
\hline & GO:0005126 & Cytokine receptor binding & $1.28 \mathrm{E}-10$ & $1.60 \mathrm{E}-08$ & IL4/TNFSF15/CASP3/STAT1/VEGFA/L10/L6/CASP8/L1B/CCL2/CXCL8/L2/IFNG/L1A/CXCL11/CXCL2/CXCL10/CD40LG & 18 \\
\hline & GO:0005125 & cytokine activity & $1.73 \mathrm{E}-10$ & $1.72 \mathrm{E}-08$ & IL4/TNFSF15/VEGFA/L10/L66/L1B/CCL2/CXCL8/L2/IFNG/LL1A/CXCL11/CXCL2/CXCL10/SPP1/CD4OLG & 16 \\
\hline & GO:0001228 & $\begin{array}{l}\text { DNA-binding transcription activator activity, } \\
\text { RNA polymerase Il-specific }\end{array}$ & $3.34 \mathrm{E}-09$ & $2.77 \mathrm{E}-07$ & PGR/AR/ESR1/RELA/RXRB/JUN/STAT1/NR1/2/NR1/3/FOS/TP63/ELK1//HIF1A/MYC/NFE2L2/PARP1/PPARA/RUNX2/E2F2/IRF1 & 20 \\
\hline & GO:0001085 & RNA polymerase II transcription factor binding & $1.80 \mathrm{E}-08$ & $1.28 \mathrm{E}-06$ & AR/ESR1/PPARG/GSK3B/JUN/AHR/FOS/RB1/ELK1/NFE2L2/PPARA/PPARD & 12 \\
\hline & GO:0020037 & Heme binding & 4.25E-08 & $2.65 \mathrm{E}-06$ & NOS2/PTGS1/PTGS2/HMOX1/CYP3A4/CYP1A2/CYP1A1/CYP1B1/DUOX2/NOS3/MPO & 11 \\
\hline & GO:0046906 & Tetrapyrrole binding & 8.89E-08 & $4.92 \mathrm{E}-06$ & NOS2/PTGS1/PTGS2/HMOX1/CYP3A4/CYP1A2/CYP1A1/CYP1B1/DUOX2/NOS3/MPO & 11 \\
\hline & GO:0019902 & Phosphatase binding & $1.28 \mathrm{E}-07$ & $6.37 \mathrm{E}-06$ & PPARG/MAPK14/SLC6A3/MET/AKT1/BCL2/STAT1/EGFR/MAPK1/SOD1/ERBB2/PPARA & 12 \\
\hline & GO:0044389 & Ubiquitin-like protein ligase binding & $1.46 \mathrm{E}-07$ & $6.60 \mathrm{E}-06$ & GSK3B/RELA/KCNH/JUN/BCL2/STAT1/EGFR/CDKN1A/RB1/NFKBIA/CASP8/HIF1A/CCNB1/CHEK2/ERBB3 & 15 \\
\hline & GO:0030374 & $\begin{array}{l}\text { Nuclear receptor transcription coactivator } \\
\text { activity }\end{array}$ & 1.63E-07 & $6.77 \mathrm{E}-06$ & NCOA2/PPARG/NCOA1/NR1/2/NR1/3/PRKCB/PPARA/PPARD & 8 \\
\hline & GO:0016705 & $\begin{array}{l}\text { Oxidoreductase activity, acting on paired } \\
\text { donors, with incorporation or reduction of } \\
\text { molecular oxygen }\end{array}$ & 2.27E- 07 & $8.71 \mathrm{E}-06$ & NOS2/PTGS1/PTGS2/HMOX1/CYP3A4/CYP1A2/CYP1A1/CYP1B1/AKR1C3/POR/NOS3 & 11 \\
\hline & GO:0048018 & Receptor ligand activity & $4.21 \mathrm{E}-07$ & 1.43E-05 & IL4/TNFSF15/VEGFA//L10/EGF/L6//L1B/CCL2/CXCL8//L2/IFNG/L1A/CXCL11/CXCL2/CXCL10/SPP1//GF2/CD40LG & 18 \\
\hline & GO:0019207 & Kinase regulator activity & 4.32E-07 & 1.43E-05 & CCNA2/CASP3/GSTP1/CCND1/CDKN1A/EGF/HSPB1//L2/CCNB1/CXCL10//GF2/ERBB3 & 12 \\
\hline & GO:0019903 & Protein phosphatase binding & $6.11 \mathrm{E}-07$ & $1.90 \mathrm{E}-05$ & PPARG/MAPK14/SLC6A3/MET/AKT1/BCL2/STAT1/EGFR/SOD1/ERBB2 & 10 \\
\hline & GO:0016209 & Antioxidant activity & $1.15 \mathrm{E}-06$ & $3.36 \mathrm{E}-05$ & PTGS1/PTGS2/GSTP1/GSTM2/SOD1/DUOX2/MPO/NQ01 & 8 \\
\hline & GO:0035257 & Nuclear hormone receptor binding & $1.30 \mathrm{E}-06$ & $3.60 \mathrm{E}-05$ & NCOA2/RXRA/ESR1/PPARG/NCOA1/STAT1/RB1/HIF1A/PRKCB/PARP1 & 10 \\
\hline & GO:0031625 & Ubiquitin protein ligase binding & 2.57E-06 & $6.75 \mathrm{E}-05$ & GSK3B/RELA/KCNH2/JUN/BCL2/EGFR/CDKN1A/RB1/NFKBIA/CASP8/HIF1A/CHEK2/ERBB3 & 13 \\
\hline & GO:0005178 & Integrin binding & $3.33 \mathrm{E}-06$ & $8.30 \mathrm{E}-05$ & KDR//CAM1/VCAM1/EGFR/PRKCA/L1B/COL3A1/SPP1//GF2 & 9 \\
\hline
\end{tabular}


Table S3 The data of KEGG pathway enrichment analysis

\begin{tabular}{|c|c|c|c|c|c|}
\hline ID & Description & P value & Padjust & Gene ID & Count \\
\hline hsa04933 & $\begin{array}{l}\text { AGE-RAGE signaling pathway in } \\
\text { diabetic complications }\end{array}$ & $1.81 \mathrm{E}-27$ & $4.37 \mathrm{E}-25$ & $\begin{array}{l}\text { MAPK14/RELA/JUN/AKT1/BCL2/BAX/CASP3/MAPK8/STAT1//CAM1/SELE/VCAM1/NEGFA/CCND1/MMP2/MAPK1/L6/PRKCA/F3/L1B/CCL2/CXCL8/PRKCB/NOS3/THBD/ } \\
\text { SERPINE1/COL1A1/L1A/COL3A1 }\end{array}$ & 29 \\
\hline hsa05418 & $\begin{array}{l}\text { Fluid shear stress and } \\
\text { atherosclerosis }\end{array}$ & $3.18 \mathrm{E}-24$ & $3.84 \mathrm{E}-22$ & $\begin{array}{l}\text { MAPK14/RELA/KDR/JUN//KBKB/AKT1/BCL2/MAPK8/HMOX1//CAM1/SELE/VCAM1/GSTP1/GSTM1/GSTM2/VEGFA/FOS/MMP2/MMP9/CAV1/L1B/CCL2/NOS3/PLAT/THBD/ } \\
\text { IFNG/L1A/NFE2L2/NQO1/CHUK }\end{array}$ & 30 \\
\hline hsa05161 & Hepatitis B & $3.73 E-22$ & $3.01 \mathrm{E}-20$ & $\begin{array}{l}\text { MAPK14/CCNA2/RELA/JUN//KBKB/AKT1/BCL2/BAX/CASP3/MAPK8/STAT1/FOS/CDKN1A/CASP9/MMP9/MAPK1/RB1/L6/ELK1/NFKBIA/CASP8/RAF1/PRKCA/MYC/CXCL8/ } \\
\text { PRKCB/BIRC5/CHUK/E2F1//E2F2 }\end{array}$ & 30 \\
\hline hsa04657 & IL-17 signaling pathway & $2.36 \mathrm{E}-21$ & $1.42 \mathrm{E}-19$ & PTGS2/MAPK14/GSK3B/RELA/JUN//L4/IKBKB/CASP3/MAPK8/MMP1/MMP3/FOS/MMP9/MAPK1/L6/NFKBIA/CASP8/L1B/CCL2/CXCL8/FNG/CXCL2/CXCL10/CHUK & 24 \\
\hline hsa05167 & $\begin{array}{l}\text { Kaposi sarcoma-associated } \\
\text { herpesvirus infection }\end{array}$ & $3.12 \mathrm{E}-21$ & $1.51 \mathrm{E}-19$ & $\begin{array}{l}\text { PTGS2/MAPK14/GSK3B/RELA/JUN//KBKB/AKT1/BAX/CASP3/MAPK8/STAT1//CAM1/PPP3CA/VEGFA/CCND1/FOS/CDKN1A/CASP9/MAPK1/RB1/L6/NFKBIA/CASP8/RAF1/ } \\
\text { HIF1A/MYC/CXCL8/CXCL2/CHUK/E2F1/E2F2 }\end{array}$ & 31 \\
\hline hsa05215 & Prostate cancer & $5.33 \mathrm{E}-21$ & $2.15 \mathrm{E}-19$ & AR/GSK3B/RELA//KBKB/AKT1/BCL2/GSTP1/MMP3/EGFR/CCND1/CDKN1A/CASP9/PLAU/MMP9/MAPK1/EGF/RB1/NFKB/A/RAF1/ERBB2/PLAT/CHUK/E2F1/E2F2 & 24 \\
\hline hsa05219 & Bladder cancer & $1.65 \mathrm{E}-19$ & $5.72 \mathrm{E}-18$ & MMP1/EGFR/VEGFA/CCND1/CDKN1A/MMP2/MMP9/MAPK1/EGF/RB1/RAF1/ERBB2/MYC/CXCL8/RASSF1/E2F1/E2F2 & 17 \\
\hline hsa04668 & TNF signaling pathway & $2.10 \mathrm{E}-19$ & $6.34 \mathrm{E}-18$ & PTGS2/MAPK14/RELA/JUN//KBKB/AKT1/CASP3/MAPK8//CAM1/SELE/VCAM1/MMP3/FOS/MMP9/MAPK1/L6/NFKBIA/CASP8/L1B/CCL2/CXCL2/CXCL10/CHUK/IRF1 & 24 \\
\hline hsa05163 & Human cytomegalovirus infection & $6.31 \mathrm{E}-19$ & $1.70 \mathrm{E}-17$ & $\begin{array}{l}\text { PTGS2/MAPK14/GSK3B/RELA//KBKB/AKT1/BAX/CASP3/PPP3CA/EGFR/VEGFA/CCND1/CDKN1A/CASP9/MAPK1/RB1/L6/ELK1/NFKBIA/CASP8/RAF1/PRKCA/MYC/L1B/ } \\
\text { CCL2/PTGER3/CXCL8/PRKCB/CHUK/E2F1/E2F2 }\end{array}$ & 31 \\
\hline hsa05212 & Pancreatic cancer & $3.23 \mathrm{E}-18$ & $7.82 \mathrm{E}-17$ & RELA//KBKB/AKT1/BAX/MAPK8/STAT1/EGFR/VEGFA/CCND1/BCL2L1/CDKN1A/CASP9/MAPK1/EGF/RB1/RAF1/ERBB2/CHUK/E2F1/E2F2 & 20 \\
\hline hsa05160 & Hepatitis C & $3.90 \mathrm{E}-18$ & 8.59E-17 & $\begin{array}{l}\text { RXRA/GSK3B/RELA//KBKB/AKT1/BAX/CASP3/STAT1/EGFR/CCND1/CDKN1A/CASP9/MAPK1/EGF/RB1/NFKBIA/CASP8/RAF1/MYC/IFNG/CLDN4/PPARA/CXCL10/CHUK/ } \\
\text { E2F1/E2F2 }\end{array}$ & 26 \\
\hline hsa05223 & Non-small cell lung cancer & 8.50E-17 & $1.71 \mathrm{E}-15$ & RXRA/RXRB/AKT1/BAX/EGFR/CCND1/CDKN1A/CASP9/MAPK1/EGF/RB1/RAF1/PRKCA/ERBB2/PRKCB/RASSF1/E2F1/E2F2 & 18 \\
\hline hsa05222 & Small cell lung cancer & $1.93 \mathrm{E}-16$ & 3.59E-15 & NOS2/PTGS2/RXRA/RELA/RXRB/KBKKB/AKT1/BCL2/BAX/CASP3/CCND1/BCL2L1/CDKN1A/CASP9/RB1/NFKBIA/MYC/CHUK/E2F1/E2F2 & 20 \\
\hline hsa01522 & Endocrine resistance & 7.19E-16 & $1.24 \mathrm{E}-14$ & ESR2/ESR1/MAPK14/JUN/AKT1/BCL2/BAX/MAPK8/EGFR/CCND1/FOS/CDKN1A/MMP2/MMP9/MAPK1/RB1/RAF1/ERBB2/E2F1/E2F2 & 20 \\
\hline hsa05142 & $\begin{array}{l}\text { Chagas disease (American } \\
\text { trypanosomiasis) }\end{array}$ & $1.64 \mathrm{E}-15$ & 2.65E-14 & NOS2/MAPK14/RELA/JUN//KBKB/AKT1/MAPK8/FOS/MAPK1/LL10/L6/NFKBIA/CASP8/L1B/CCL2/CXCL8/L2/SERPINE1//FNG/CHUK & 20 \\
\hline hsa05225 & Hepatocellular carcinoma & $3.79 \mathrm{E}-15$ & $5.73 \mathrm{E}-14$ & GSK3B/MET/AKT1/BAX/HMOX1/GSTP1/GSTM1/GSTM2/EGFR/CCND1/BCL2L1/CDKN1A/MAPK1/RB1/ELK1/RAF1/PRKCA/MYC/PRKCB/NFE2L2/NQO1/E2F1/E2F2//GF2 & 24 \\
\hline hsa04659 & Th17 cell differentiation & 4.37E-15 & $6.22 \mathrm{E}-14$ & RXRA/MAPK14/RELA/RXRB/JUN/L4/IKBKB/MAPK8/STAT1/AHR/PPP3CA/FOS/MAPK1/L6/NFKBIA/HIF1A/L1LB/L2/IFNG/CHUK & 20 \\
\hline hsa04066 & HIF-1 signaling pathway & $6.36 \mathrm{E}-15$ & 8.55E-14 & NOS2/RELA/AKT1/BCL2/HMOX1/INSR/EGFR/VEGFA/CDKN1A/MAPK1/EGF/L6/PRKCA/HIF1A/ERBB2/PRKCB/NOS3/SERPINE1//FNG/HK2 & 20 \\
\hline hsa05169 & Epstein-Barr virus infection & $2.60 \mathrm{E}-14$ & $3.31 \mathrm{E}-13$ & $\begin{array}{l}\text { MAPK14/CCNA2/RELA/JUN//KBKB/AKT1/BCL2/BAX/CASP3/MAPK8/STAT1//CAM1/PSMD3/CCND1/CDKN1A/CASP9/RB1/L6/NFKBIA/CASP8/MYC/CXCL10/CHUK/E2F1/ } \\
\text { E2F2 }\end{array}$ & 25 \\
\hline hsa04625 & $\begin{array}{l}\text { C-type lectin receptor signaling } \\
\text { pathway }\end{array}$ & 3.44E-14 & 4.17E-13 & PTGS2/MAPK14/RELA/JUN//KBKB/AKT1/MAPK8/STAT1/PPP3CA/MAPK1/LL10/L6/NFKBIA/CASP8/RAF1//L1B/L2/CHUK/IRF1 & 19 \\
\hline
\end{tabular}

